ARTICLE

\title{
Van der Waals interfacial reconstruction in monolayer transition-metal dichalcogenides and gold heterojunctions
}

\author{
Ruichun Luo (1) 1,2,6, Wen Wu Xu 3,6, Yongzheng Zhang ${ }^{4}$, Ziqian Wang ${ }^{2,4}$, Xiaodong Wang ${ }^{1}$, Yi Gao (i) ${ }^{5}$, \\ Pan Liu ${ }^{1,4 凶} \&$ Mingwei Chen ${ }^{2,4 凶}$
}

The structures and properties of van der Waals (vdW) heterojunctions between semiconducting two-dimensional transition-metal dichalcogenides (2D TMDs) and conductive metals, such as gold, significantly influence the performances of 2D-TMD based electronic devices. Chemical vapor deposition is one of the most promising approaches for large-scale synthesis and fabrication of 2D TMD electronics with naturally formed TMD/metal vdW interfaces. However, the structure and chemistry of the vdW interfaces are less known. Here we report the interfacial reconstruction between TMD monolayers and gold substrates. The participation of sulfur leads to the reconstruction of $\mathrm{Au}\{001\}$ surface with the formation of a metastable $\mathrm{Au}_{4} \mathrm{~S}_{4}$ interfacial phase which is stabilized by the top $\mathrm{MoS}_{2}$ and $\mathrm{WS}_{2}$ monolayers. Moreover, the enhanced vdW interaction between the reconstructed $\mathrm{Au}_{4} \mathrm{~S}_{4}$ interfacial phase and TMD monolayers results in the transition from n-type TMD-Au Schottky contact to $\mathrm{p}$ type one with reduced energy barrier height.

\footnotetext{
${ }^{1}$ Shanghai Key Laboratory of Advanced High-temperature Materials and Precision Forming, State Key Laboratory of Metal Matrix Composites, School of Materials Science and Engineering, Shanghai Jiao Tong University, Shanghai 200240, P. R. China. ${ }^{2}$ Department of Materials Science and Engineering, Johns Hopkins University, Baltimore, MD 21218, USA. ${ }^{3}$ Department of Physics, School of Physical Science and Technology, Ningbo University, Ningbo 315211, P. R. China. ${ }^{4}$ WPI Advanced Institute for Materials Research, Tohoku University, Sendai 980-8577, Japan. ${ }^{5}$ Shanghai Advanced Research Institute, Chinese Academy of Sciences, Shanghai 201210, P. R. China. ${ }^{6}$ These authors contributed equally: Ruichun Luo, Wen Wu Xu. ${ }^{凶}$ email: panliu@sjtu.edu.cn; mwchen@jhu.edu
} 
S emiconducting 2D TMDs, such as $\mathrm{MoS}_{2}$ and $\mathrm{WS}_{2}$, have shown exceptional electronic ${ }^{1}$, optical ${ }^{2}$, and catalytic $^{3}$ properties superior to their bulk counterparts. To employ $2 \mathrm{D}$ TMDs in electronic devices, one of the essential processes is to connect the atomically-thick crystals with bulk metals that serve as electrodes, connectors and supporting substrates ${ }^{4-6}$. The structure and chemistry of $2 \mathrm{D}$ semiconductor-metal interfaces significantly influence the performances of $2 \mathrm{D}$ electronic devices ${ }^{7}$. Two types of contacts, covalent and vdW interfaces from Ti$\mathrm{MoS}_{2}$ and $\mathrm{Au}-\mathrm{MoS}_{2}$, have been suggested by previous theoretical invetigations ${ }^{8,9}$. However, it has been found that both interfaces encounter the fermi-level pinning ${ }^{10}$ and, thus, performance degradation of the heterostructures that are fabricated by depositing or lithographing metals onto 2D TMDs ${ }^{11,12}$. Atomic scale characterization of the interfaces by scanning transmission electron microscopy (STEM) has suggested that the degradation is associated with the damage of TMDs in the contact layer by invasion of metal atoms ${ }^{12-14}$. Interface engineering, such as insertion of additional vdW layers (graphene $\mathrm{e}^{15}$, hexagonal boron nitride $(\mathrm{hBN})^{16}$, etc.), mechanical transfer of metal films ${ }^{13}$ and incorporation with indium ${ }^{17}$, are explored to form ideal vdW contacts that are free from the chemical disorder.

On the other hand, chemical vapor deposition (CVD) is a welldeveloped method to grow high quality $2 \mathrm{D}$ materials and to fabricate electronic devices on various substrates ${ }^{18}$, which provides the possibility to directly obtain perfect vdW contacts with metals, like graphene grown on $\mathrm{Cu}$ (111) surface $^{19}$ and hBN grown on $\mathrm{Cu}(110)$ surface $^{20}$. As to the CVD growth of TMDs, insulating substrates are commonly used ${ }^{21}$ and $\mathrm{Au}$ is almost the only metal substrate and is known to form vdW interfaces with TMDs thanks to its chemical stability in chalcogen-rich environments during $\mathrm{CVD}^{22-25}$. Although Au is inert in bulk form, $\mathrm{Au}$ surface atoms has rich ligand chemistry partially due to the existence of possible oxidation states of gold. Various Au-thiolate complexes and low-temperature chemisorbed sulfur have been observed on Au surfaces ${ }^{26,27}$. Accordingly, the vapored sulfur and S-Mo precursors formed during CVD reactions may affect the atomic structures of gold surfaces and, subsequently, alter interfacial interactions with as-deposited 2D TMDs. Experimentally, enhanced electrocatalytic activities and quenched photoluminescence from possible electron coupling have been observed from monolayer $\mathrm{MoS}_{2}$ grown on gold substrates ${ }^{22,23,28}$. In addition, $\mathrm{Au}$ substrates are found to arouse the $1 \mathrm{H}-1 \mathrm{~T}$ phase transformation of $\mathrm{MoS}_{2}$ monolayers by inducing the in-plane shifting of $\mathrm{S}$ or/and Mo atom layers ${ }^{22,29,30}$. The interaction between monolayer $\mathrm{MoS}_{2}$ and gold substrates has been investigated by scanning tunneling microscope (STM) and periodically striped and moiré superstructures, most likely rising from the large lattice mismatch between the basal plane of $\mathrm{MoS}_{2}$ and $\mathrm{Au}$ $(001)$ or $(111)^{31,32}$, have been observed from the planar view. In contrast, the cross-sectional views, which can directly present the structure and chemistry of interfaces, have not been realized in the CVD grown TMD/Au junctions.

In this study, we report atomic-scale observations of the interfaces between $\mathrm{Au}$ and monolayer $\mathrm{MoS}_{2}$ and $\mathrm{WS}_{2}$ from crosssectional views by growing the monolayer TMDs on 3D nanoporous gold (NPG). NPG has been demonstrated as an effective $3 \mathrm{D}$ substrate for CVD growth of TMDs ${ }^{22,28}$. The large surface area and high conductivity of NPG can dramatically enhance the electrocatalysis and photoelectrocatalysis of 2D TMDs. More importantly, plentiful facets with different crystallographic orientations of NPG offer a unique opportunity to directly observe the cross-sectional structures of the monolayer $\mathrm{MoS}_{2}$ on different surfaces of $\mathrm{Au}$ without additional TEM sample preparation, such as focused ion beam (FIB) milling and ion milling which may introduce unexpected interface damage. The pristine
$\mathrm{TMD} / \mathrm{Au}$ interfaces well preserve the structural integrity and show reconstructed interfaces with the formation of an unconventional $\mathrm{Au}_{4} \mathrm{~S}_{4}$ superstructure on the topmost surface of $\mathrm{Au}$ $\{100\}$. Moreover, the enhanced vdW interaction between $\mathrm{MoS}_{2}$ and reconstructed $\mathrm{Au}$ surfaces leads to the transition from n-type TMD-Au Schottky contact to p-type one with reduced barrier height.

\section{Results}

Synthesis and characterization of monolayer TMDs on NPG. $\mathrm{MoS}_{2}$ was grown on a $100 \mathrm{~nm}$ thick NPG film, which was prepared by chemical dealloying ${ }^{33}$, by a low-pressure CVD method (Supplementary Fig. 1) ${ }^{34}$. The high CVD temperature of $923 \mathrm{~K}$ leads to the slight coarsening of nanoporous structure of NPG from the as-dealloyed pore size of $\sim 20$ to $\sim 50 \mathrm{~nm}$ (Supplementary Fig. 2). Meanwhile, the coarsening also leads to the development of multiple low-energy surface facets ${ }^{35}$ for cross-sectional TEM observations. Figure la shows the high angle annual dark-field (HAADF) STEM image of NPG covered by a thin layer of $\mathrm{MoS}_{2}$. By carefully optimizing the CVD conditions, monolayer $\mathrm{MoS}_{2}$ can be obtained, which is evidenced by STEM images (Fig. 1b), as well as Raman spectra (Fig. 2a) with a narrowed frequency difference $\left(\Delta \sim 20 \mathrm{~cm}^{-1}\right.$ compared to the multi-layered value of $\left.\sim 25 \mathrm{~cm}^{-1}\right)$ between $E^{1}{ }_{2 \mathrm{~g}}\left(\sim 384 \mathrm{~cm}^{-1}\right)$ and $A_{1 \mathrm{~g}}\left(\sim 404 \mathrm{~cm}^{-1}\right)^{36}$. X-ray photoemission spectroscopy (XPS) measurements (Fig. 2b, c) were also performed to characterize the formation of $\mathrm{MoS}_{2}$. The XPS spectra show the Mo $3 d_{5 / 2}(229.1 \mathrm{eV})$ and Mo $3 d_{3 / 2}(232.2 \mathrm{eV})$ peaks from $\mathrm{Mo}^{4+}$ as well as the S $2 p_{3 / 2}(161.9 \mathrm{eV})$ and S $2 p_{1 / 2}(163.1 \mathrm{eV})$ peaks from $\mathrm{S}^{2-}$ (ref. ${ }^{23}$ ), indicating the formation of semiconducting $2 \mathrm{D}$ $\mathrm{MoS}_{2}$ (2H phase for multilayer and/or $1 \mathrm{H}$ phase for monolayer). By comparing with the XPS results of $\mathrm{MoS}_{2}$ grown on the glass substrate, large energy shifts of the Mo $3 d$ and S $2 p$ peaks of $\mathrm{MoS}_{2}$ on NPG can be seen. For Mo $3 d_{5 / 2}$ and Mo $3 d_{3 / 2}$ core levels, the peaks shift from 230.3 and $233.4 \mathrm{eV}$ of $\mathrm{MoS}_{2}$ on glass to 229.1 and $232.2 \mathrm{eV}$ of $\mathrm{MoS}_{2}$ on NPG. For S $2 p_{3 / 2}$ and S $2 p_{1 / 2}$ core levels, the binding energies shift from 163.1 and $164.3 \mathrm{eV}$ of $\mathrm{MoS}_{2}$ on glass to 161.9 and $163.1 \mathrm{eV}$ of $\mathrm{MoS}_{2}$ on NPG. The obvious binding energy changes imply an enhanced interaction between $\mathrm{MoS}_{2}$ and $\mathrm{Au}$ in comparison with $\mathrm{MoS}_{2}$ and glass. It is worth noting that the S $2 p$ doublet peaks of $\mathrm{MoS}_{2}$ on NPG become broad and exhibit weak shoulders, marked by arrows, compared to those of $\mathrm{MoS}_{2}$ on glass. Correspondingly, the doublet peaks of $\mathrm{Au} 4 f$ from NPG also become broad and asymmetric with a slightly blue shift of $0.3 \mathrm{eV}$ after the growth of $\mathrm{MoS}_{2}$ (Fig. 2d), indicating the possible formation of $\mathrm{Au}-\mathrm{S}$ bonding in the $\mathrm{MoS}_{2}$ on NPG system. Moreover, we performed the quantitative XPS analysis of the composition of $\mathrm{MoS}_{2}$ grown on glass and NPG. The corresponding quantifications of Mo and $\mathrm{S}$ in atomic \% and their ratios from three independent measurements are shown in Supplementary Table 1. For the samples of $\mathrm{MoS}_{2}$ on glass, the $\mathrm{Mo} / \mathrm{S}$ ratios are all larger than 0.5 , the stoichiometric ratio of $\mathrm{MoS}_{2}$, which is consistent with previous reports that CVD grown $\mathrm{MoS}_{2}$ contains sulfur vacancies $^{37-39}$. However, for the samples of $\mathrm{MoS}_{2}$ on NPG, the Mo/S ratios are all less than 0.5 , suggesting the existence of excess sulfur in the system. Combined with the facts that the covalent bond between gold and sulfur could form ${ }^{26,27}$, the XPS results suggest the possible formation of the $\mathrm{Au}-\mathrm{S}$ bonding after $\mathrm{MoS}_{2}$ growing on NPG.

The multiple terminal surfaces with different facets of NPG provide various $\mathrm{Au}$-TMDs interfaces for direct cross-sectional observations ${ }^{40,41}$. A high resolution HAADF-STEM image (Fig. 1b) viewed along the $\mathrm{Au}[110]\left([110]_{\mathrm{Au}}\right)$ direction shows an intact $\mathrm{MoS}_{2}$ monolayer attached on the gold surface with different facets, indicating the uniform growth of $\mathrm{MoS}_{2}$ on the internal surface of NPG. The zoom-in images of Au (001) surface 

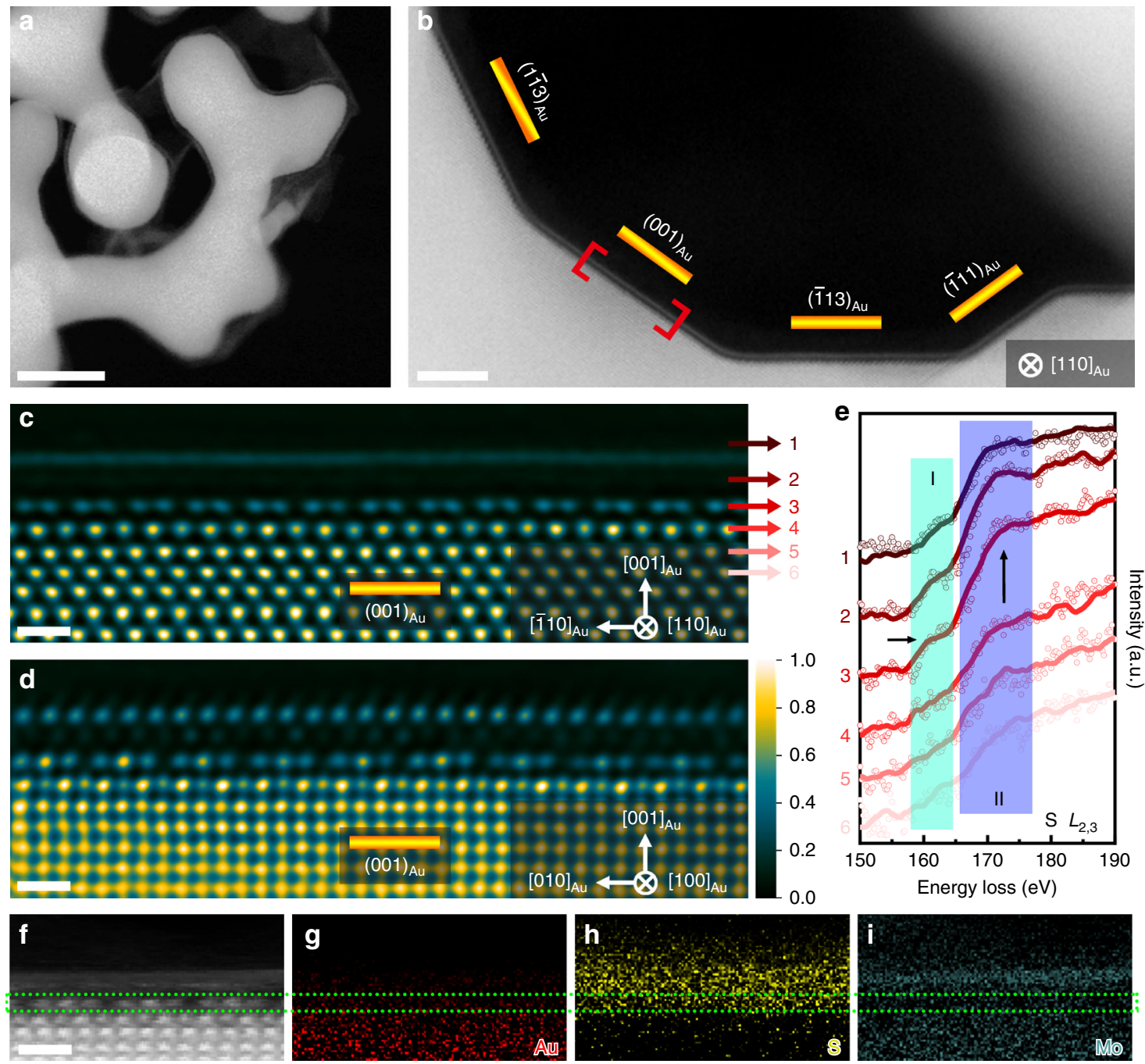

Fig. 1 Structural and chemical characterization of the interface between monolayer $\mathbf{M o S}_{\mathbf{2}}$ and NPG. a Low magnification HAADF-STEM image of the $\mathrm{MoS}_{2}$ film grown on NPG. Scale bar, $50 \mathrm{~nm}$. b Magnified HAADF-STEM image of monolayer $\mathrm{MoS}_{2}$ grown on the internal surfaces of NPG. Scale bar, $5 \mathrm{~nm}$. c, d Filtered HAADF-STEM images viewed from the $[110]_{\mathrm{Au}}(\mathbf{c})$ and $[100]_{\mathrm{Au}}(\mathbf{d})$ directions. Color variation from black to blue and then to yellow corresponds to the intensity from low to high. Scale bar, $0.5 \mathrm{~nm}$. e EELS spectra of $S L_{2,3}$ core-level edges summed from the different layers of MoS ${ }_{2}$ and $A u$

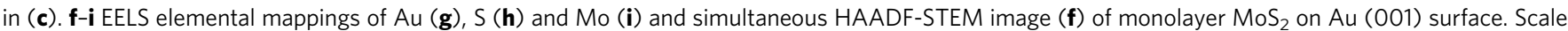
bar, $0.5 \mathrm{~nm}$.

along both the $[110]_{\mathrm{Au}}$ and $[100]_{\mathrm{Au}}$ directions (Fig. 1c, d) reveal the atomically sharp $\mathrm{Au}-\mathrm{MoS}_{2}$ contact as well as the arrangements of $\mathrm{S}$ atoms in the semiconducting $1 \mathrm{H} \mathrm{MoS}$ monolayer. Interestingly, an unusual periodic structure, different from the face-centered cubic (FCC) order of $\mathrm{Au}$ and $1 \mathrm{H}$ of $\mathrm{MoS}_{2}$, can be observed from the $\mathrm{Au}$ (001) surface underneath the monolayer $\mathrm{MoS}_{2}$ (Fig. 1c, d), indicating the occurrence of interfacial reconstruction during $\mathrm{MoS}_{2}$ growth. From the $[110]_{\mathrm{Au}}$ imaging direction (Fig. 1c), the periodic structure of the topmost $\mathrm{Au}$ (001) layer consists of paired atom columns with a lower intensity compared to the FCC matrix. Moreover, the visible intensity variation in a periodic manner can also be observed from the sublayer (the second Au atom layer counting from the topmost surface layer of $\mathrm{Au}$ ) although the projected atomic occupations from the sublayer columns still locate at the FCC lattice. Since the intensity of each column is proportional to the Au atom number in the column, the periodic intensity variation indicates a regular change of $\mathrm{Au}$ atom numbers in the sublayer and thus a different ordering sequence. Consequently, the top two layers of the $\mathrm{Au}$ (001) surface experience a structural reconstruction during the CVD growth. From $[100]_{\mathrm{Au}}$ imaging direction (Fig. 1d), the interfacial reconstruction can be directly visualized as the topmost surface of $\mathrm{Au}$ (001) shows a trios structure in which the center atom columns have a contrast close to the FCC Au matrix and two side atom columns have a relatively weaker contrast. Meanwhile, the sublayer atom columns, viewed along $[100]_{\mathrm{Au}}$ direction, also show the reconstructed periodic intensity variation which is similar to that viewed from $[110]_{\mathrm{Au}}$ direction. The intensity line profiles of these two reconstructed $\mathrm{Au}$ layers, displaying the periodic ordering and intensity variation, can be seen in Fig. 3, together with the raw HAADF-STEM images. To the best of our knowledge, the distinctive structural periodicity at the interface between $\mathrm{Au}(001)$ and $\mathrm{MoS}_{2}$ monolayer has not been observed from reconstructed $\mathrm{Au}$ (001) surfaces before. Importantly, the same interfacial reconstruction can be observed from all the $\mathrm{Au}$ (001) facets covered by $1 \mathrm{H} \mathrm{MoS}$ in this study (Supplementary Fig. 3), demonstrating the universality of the Au (001) surface reconstruction under $1 \mathrm{H} \mathrm{MoS}_{2}$.

The chemistry of the reconstructed $\mathrm{Au}$ (001) surface under $\mathrm{MoS}_{2}$ was investigated by electron energy loss spectroscopy (EELS) with an atomic-scale spatial resolution under STEM mode. Figure 1e is the summed EELS spectra of $S L_{2,3}$ core-level 

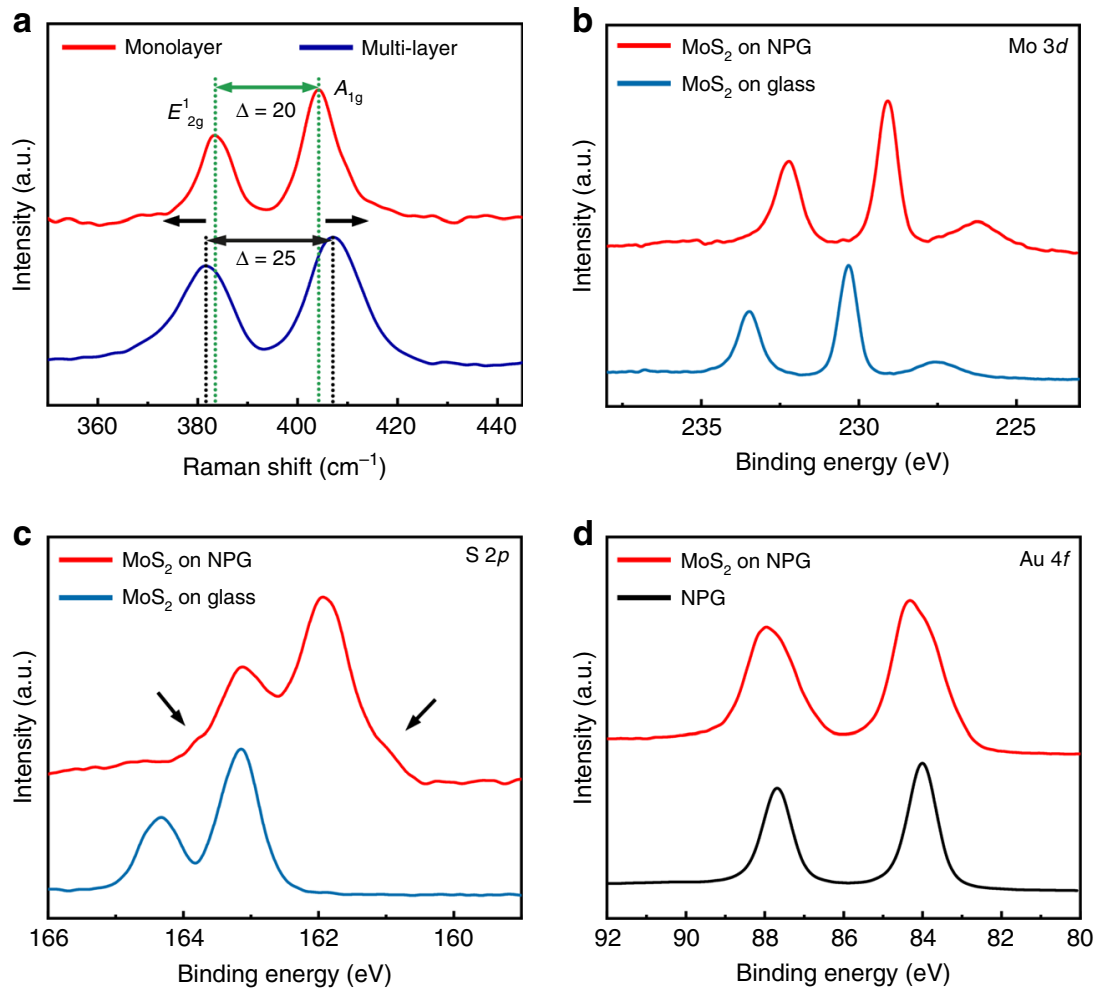

Fig. 2 Raman and XPS characterizations of $\mathbf{M o S}_{\mathbf{2}}$ growing on NPG. a Raman spectra of monolayer and multi-layer MoS on NPG. b Mo $3 d$ and $\mathbf{c} S 2 p$ core-level peaks of $\mathrm{MoS}_{2}$ grown on NPG and glass. d Au $4 f$ core-level peaks of original NPG and $\mathrm{MoS}_{2}$ grown on NPG.
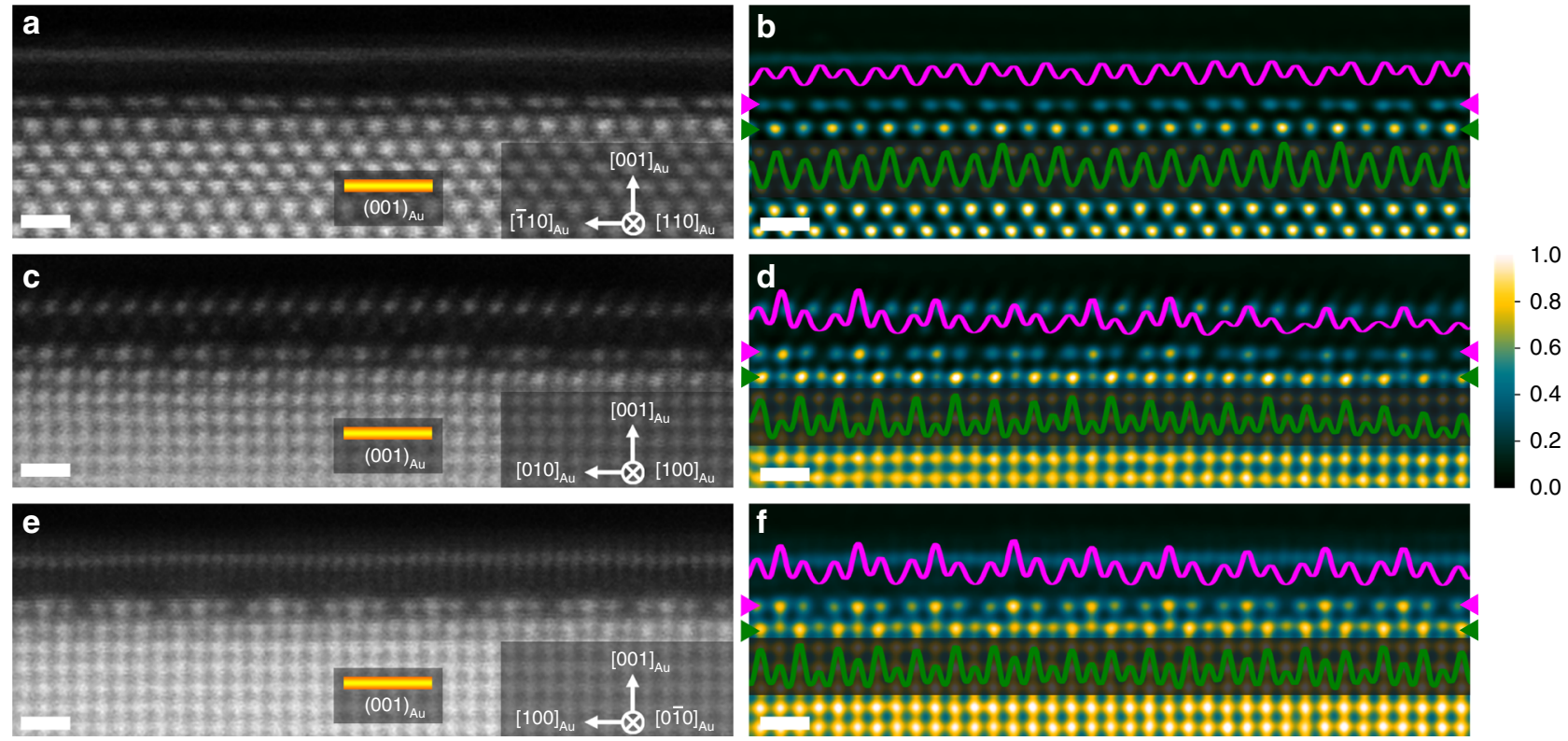

Fig. 3 Structural characterizations of the interfaces between $\mathbf{M o S}_{\mathbf{2}}$ monolayer and Au (001) surface of NPG. a, c, e Raw HAADF-STEM images viewed from the $[110]_{\mathrm{Au}}(\mathbf{a}),[100]_{\mathrm{Au}}(\mathbf{c})$ and $[0 \overline{10}]_{\mathrm{Au}}(\mathbf{e})$ directions. Scale bar, $0.5 \mathrm{~nm}$. b, d, f Filtered atomic-resolution HAADF-STEM images with false color viewed from the $[110]_{\mathrm{Au}}(\mathbf{b}),[100]_{\mathrm{Au}}(\mathbf{c})$ and $[0 \overline{10}]_{\mathrm{Au}}(\mathbf{e})$ directions. Color variation from black to blue and then to yellow corresponds to the intensity from low to high. The magenta and green lines are intensity profiles measured along the Au topmost layer and sublayer following the corresponding arrows in (b, d) and (f), respectively. Scale bar, $0.5 \mathrm{~nm}$.

edges acquired from $\mathrm{MoS}_{2}$ to Au by layer-by-layer analysis. The dominating features of the $S L_{2,3}$ fine structure in reign I and II is composed of S $3 \mathrm{~s}$ and $3 \mathrm{~d}$ partial density of states (DOS) ${ }^{42}$. The reconstructed topmost Au layer (Layer \#3) shows a strong $\mathrm{S}$ signal, which is distinct from the lower layers (\#4, \#5, and \#6) and suggests that sulfur is involved into the topmost surface reconstruction. In addition, the atomic-scale EELS measurements also reveal the elemental distributions of $\mathrm{Au}, \mathrm{Mo}$ and $\mathrm{S}$ in the $\mathrm{Au}$ $\mathrm{MoS}_{2}$ interface with monolayer $\mathrm{MoS}_{2}$ grown on $\mathrm{Au}$ (001) surface (Fig. 1g-i, Supplementary Fig. 4), together with simultaneous HAADF-STEM imaging (Fig. 1f), further confirming that the reconstructed $\mathrm{Au}(001)$ topmost surface is constituted by $\mathrm{Au}$ and 


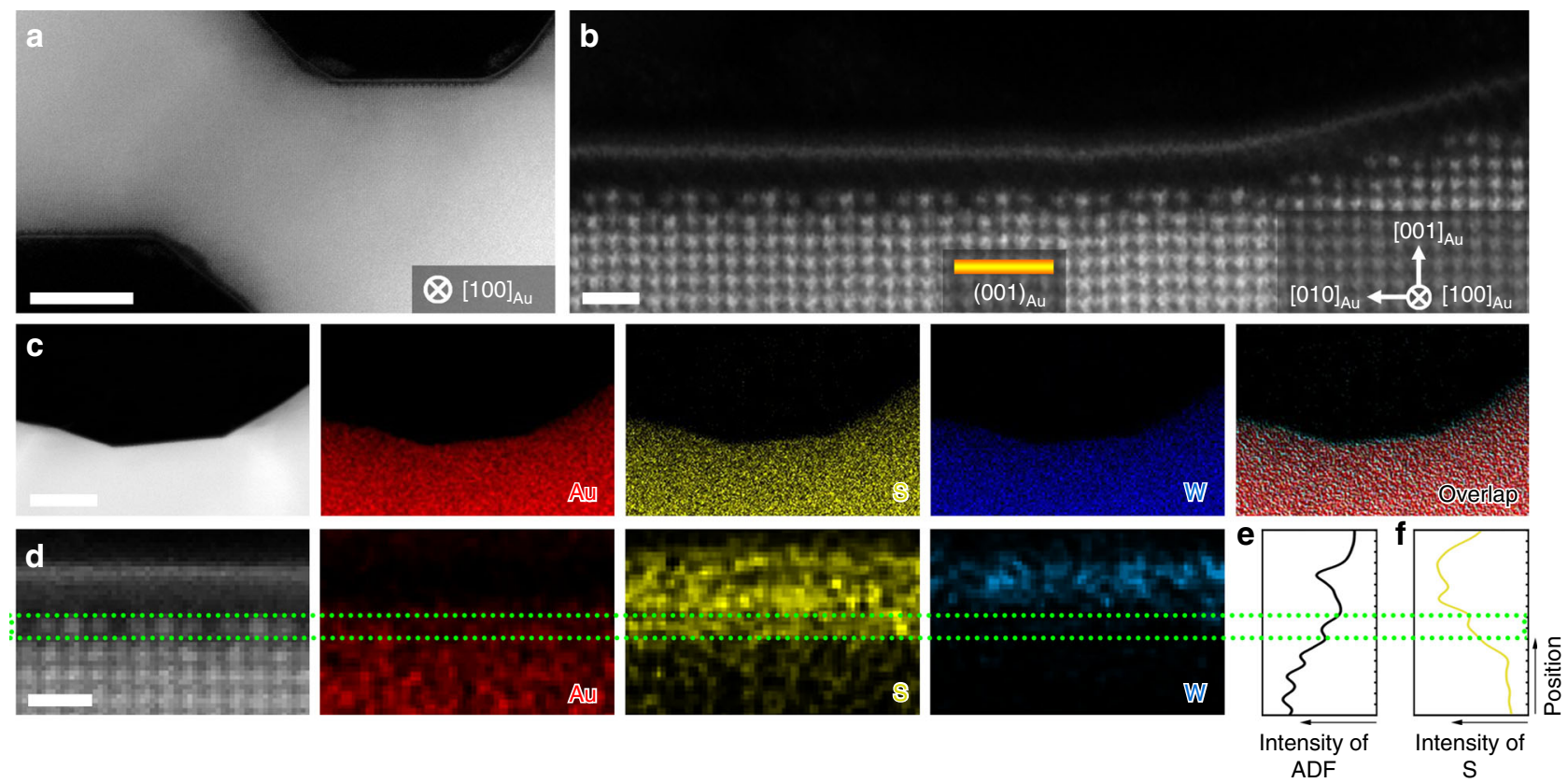

Fig. 4 Structural and chemical characterizations of the interface between monolayer $\mathbf{W S}_{\mathbf{2}}$ and $\mathbf{N P G}$. a HAADF-STEM image of the monolayer WS 2 grown on NPG. Scale bar, $10 \mathrm{~nm}$. b Atomic-resolution HAADF-STEM image of monolayer $\mathrm{WS}_{2}$ grown along the NPG surfaces of NPG. Scale bar, $0.5 \mathrm{~nm}$. c EDS elemental mappings and simultaneously HAADF-STEM image of $\mathrm{WS}_{2}$ on Au (001). Scale bar, $50 \mathrm{~nm}$. d EELS elemental mappings and simultaneously HAADF-STEM image of monolayer $\mathrm{WS}_{2}$ grown on $\mathrm{Au}(001)$ surface. Scale bar, $0.5 \mathrm{~nm}$. e, $\mathbf{f}$ Intensity profiles measured from the HAADFSTEM image (e) and S EELS mapping (f).

$S$ elements. However, it appears that sulfur does not invade into the sublayer of $\mathrm{Au}(001)$ surface since the EELS spectrum of Layer \#4 is similar to Layer \#5 and \#6 (Fig. 1e) where $S$ cannot be detected.

Besides $\mathrm{MoS}_{2}$, we also found that the interfacial reconstruction occurs in the contact between monolayer $\mathrm{WS}_{2}$ and NPG. As shown in Fig. 4a, the internal surface of NPG is covered by monolayer $\mathrm{WS}_{2}$, which is grown by the similar CVD process. The same surface reconstruction of $\mathrm{Au}(001)$ can be observed from the atomic-scale HAADF-STEM image viewed from $[110]_{\mathrm{Au}}$ direction (Fig. 4b). The direct comparison of the reconstructed $\mathrm{Au}$ (001) surfaces under $\mathrm{MoS}_{2}$ and $\mathrm{WS}_{2}$ is shown in Supplementary Fig. 5. The chemical distribution from energy disperse spectroscopy (EDS) elemental mappings confirms the heterostructure of monolayer $\mathrm{WS}_{2}$ lying on the $\mathrm{Au}$ surface (Fig. 4c). Moreover, the EELS mappings reveal the refined chemical information that the reconstructed interfacial phase consists of $\mathrm{Au}$ and $\mathrm{S}$ atoms (Fig. 4d). The intensity profiles measured from the HAADFSTEM image and $S L_{2,3}$ EELS mapping along the out-of-plane direction are shown in Fig. $4 \mathrm{e}, \mathrm{f}$, confirming that $\mathrm{S}$ atoms also involve into the structural reconstruction of the $\mathrm{Au}$ topmost surface in the $\mathrm{WS}_{2}-\mathrm{NPG}$ system.

We also investigated the interfaces between monolayer TMD films and other Au surfaces with different crystallographic orientations. The interfacial reconstructions have been observed from $\mathrm{Au}\{110\},\{111\},\{012\}$, and $\{113\}$ surfaces. Again, sulfur can be detected from the reconstructed Au topmost surfaces by EELS spectra and elemental mappings (Supplementary Fig. 6). The atomic structures of these reconstructed interfaces appear different from that of $\mathrm{Au}\{100\}$. The details of the structural characterization and modeling will be discussed in a separate paper.

Structural determination of the reconstructed $\mathrm{MoS}_{2} / \mathrm{Au}\{001\}$ interface. The intensity of an atomic column in HAADF-STEM images is proportional to $\sim Z^{n}$, where $Z$ denotes atomic number ${ }^{43}$ and $n$ is a constant in the range $1.6-1.9$ for most cases ${ }^{44}$. The contrast of $\mathrm{S}(Z=16)$ residing at the reconstructed $\mathrm{Au}(001)$ surface is too low, in comparison with $\mathrm{Au}(Z=79)$, to be identified from the HAADF-STEM images. Thus, the image contrast of the atomic columns at the $\mathrm{Au}\{001\}$ topmost surface is dominantly from $\mathrm{Au}$ atoms, and the relatively dark contrast represents fewer $\mathrm{Au}$ atoms in the columns. Based on the HAADF-STEM projections along both $[110]_{\mathrm{Au}}$ and $[100]_{\mathrm{Au}}$ directions, we can deduce that the atomic arrangements of the topmost $\mathrm{Au}$ atoms exhibit a square-octagon lattice from the top view of the reconstructed Au (001) surface (Fig. 5). Each vertex of the square lattice is decorated with a tilted square ${ }^{45}$. This structure has been predicted to be a topological phase ${ }^{46}$ or quantum magnetic phase ${ }^{47}$ by density functional theory (DFT) calculations. As to the Au (001) sublayer, it is only composed by Au atoms and the columns with weaker contrast represent the periodic absence of $\mathrm{Au}$ atoms (Supplementary Fig. 7b).

To locate the occupation of $\mathrm{S}$ atoms in the reconstructed $\mathrm{Au}$ $\{001\}$ surface, we performed annular bright-field (ABF) STEM observations (Fig. 6). Additional weak contrast from S can be recognized at the gap between two pairs of $\mathrm{Au}$ atomic columns from the ABF-STEM image taken along the $[110]_{\mathrm{Au}}$ direction as well as the corresponding intensity line profile. On the contrary, no extra contrast can be detected from $[100]_{\mathrm{Au}}$ ABF-STEM images because $\mathrm{S}$ atoms overlap with $\mathrm{Au}$ atoms in the atomic columns along the $[100]_{\mathrm{Au}}$ direction. Combining the fact that the $\mathrm{Au}-\mathrm{S}$ bonds are commonly realized as staple-like $\mathrm{S}-\mathrm{Au}-\mathrm{S}$ linear structures at thiolate-protected gold surfaces and interfaces ${ }^{26,48}$, the reconstructed $\mathrm{Au}\{001\}$ surface can be assembled by planar $\mathrm{Au}_{4} \mathrm{~S}_{4}$ square rings shown in the dotted box of Fig. $7 \mathrm{~g}$, which has been observed in sulfur-containing metalloid gold clusters ${ }^{49}$. This surface atomic configuration gives the best match with the HAADF- and ABF-STEM images in both $[110]_{\mathrm{Au}}$ and $[100]_{\mathrm{Au}}$ directions, as well as the chemical analyses from XPS and EELS.

To verify and optimize the reconstructed surface structure of $\mathrm{Au}\{001\}$ derived from the STEM observations, we conducted DFT calculations (see the Methods section). The primary 


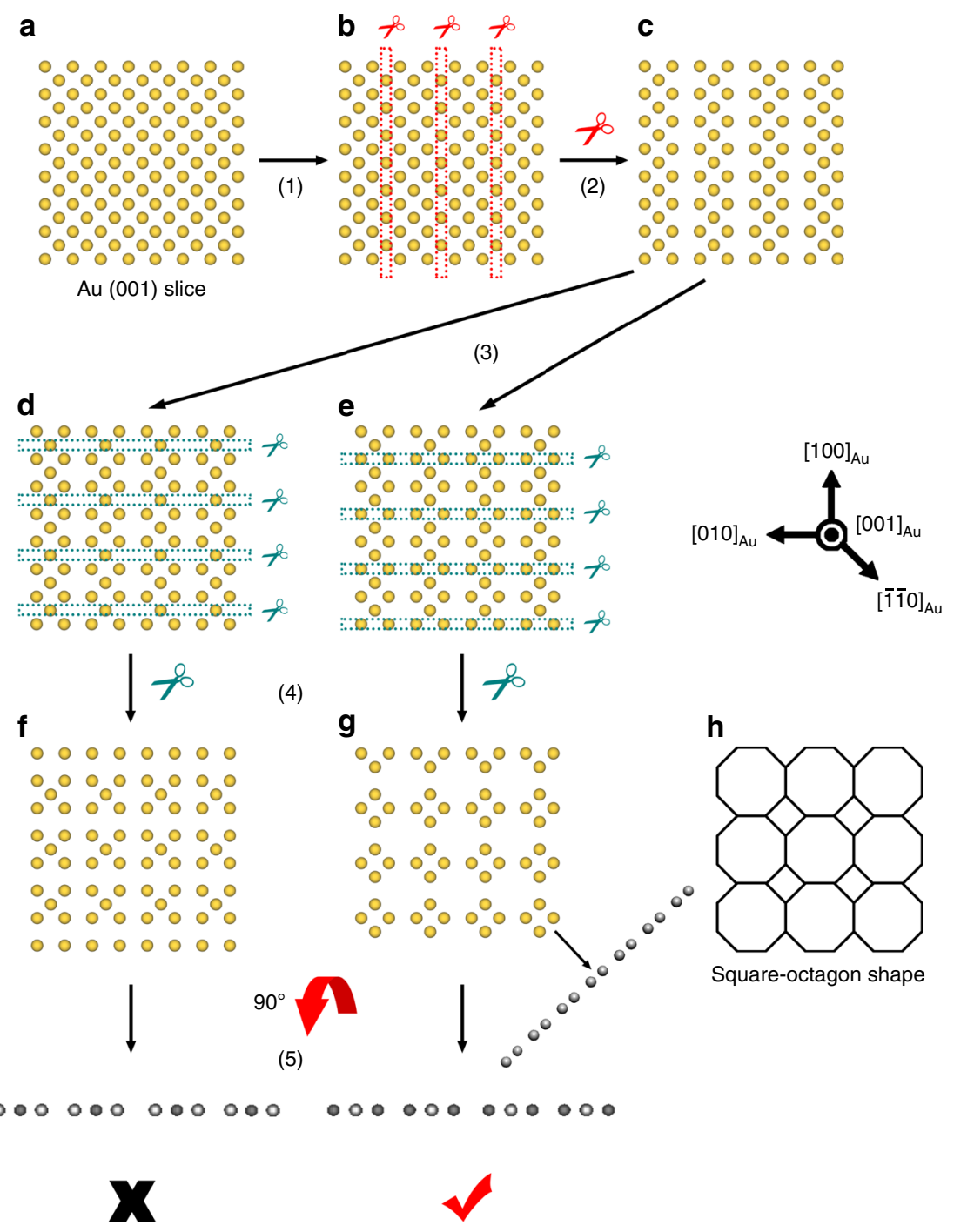

Fig. 5 Structural determination of Au atom position in the reconstructed Au (001) surface on the basis of the HAADF-STEM images. a A monolayer Au (001) slice viewed from $\mathrm{Au}$ [001] direction. b Deleting the atoms in one column every three columns to make the gaps meet the periodicity viewed from Au [100] direction. $\mathbf{c}$ The structure with remaining Au atoms after process in (b). $\mathbf{d}$, e Two different ways to delete the atoms in one row every three rows to make the gaps meet the periodicity viewed from Au [100] direction. $\mathbf{f}$ The structure with remaining Au atoms after process in (d), which is not consistent with the experimental HAADF-STEM images. $\mathbf{g}$ The structure with remaining Au atoms after process in (e), which is consistent with the experimental HAADF-STEM images in every directions we have observed. $\mathbf{h}$ The square-octagon structure composed by the remaining monolayer Au atoms.

structural models were based on the HAADF-STEM images with the orientation relationship between $\mathrm{Au}$ and monolayer $\mathrm{MoS}_{2}$ of $(001)_{\mathrm{Au}} / /(001)_{\mathrm{MoS} 2}$ and $[100]_{\mathrm{Au}} / /[010]_{\mathrm{MoS} 2}$, which is commonly observed in our study. After relaxation, the lattice mismatch is only $1.77 \%$ between monolayer $\mathrm{MoS}_{2}$ and $\mathrm{Au}_{4} \mathrm{~S}_{4}$. The DFToptimized structure viewed from $[110]_{\mathrm{Au}}$ and $[010]_{\mathrm{Au}}$ directions are shown in Fig. $7 \mathrm{a}, \mathrm{d}$, respectively. The simulated HAADF images (Fig. 7b, e) from corresponding directions of the structure model are in remarkable consistency with the experimental ones (Fig. 7c, f). Quantitative image intensity analyses (Fig. 7h, i) of the $\mathrm{Au}_{4} \mathrm{~S}_{4}$ monolayers and $\mathrm{Au}$ sublayers from the experimental (solid lines with different color) and simulated images (dashed lines) also shows good agreement from both projection directions. Moreover, the distance of $2.67 \AA$ between the $\mathrm{Au}_{4} \mathrm{~S}_{4}$ monolayer and the bottom $\mathrm{S}$ layer of $\mathrm{MoS}_{2}$ is also close to the experimental measurement $(\sim 2.56 \AA)$. Figure $7 \mathrm{~g}$ exhibits the top view of the reconstructed $\mathrm{Au}$ (001) surface with the formation of $\mathrm{Au}_{4} \mathrm{~S}_{4}$ ring motifs. Its orientation relations with the Au matrix, sublayer, and $\mathrm{MoS}_{2}$ layer are shown in Supplementary Fig. 7.
Factors influencing the interfacial $\mathrm{Au}_{4} \mathrm{~S}_{4}$ phase formation. There is a strict co-existence relation between the reconstructed $\mathrm{Au}(001)$ surfaces and 2D TMDs. The surface reconstruction only takes place at the $2 \mathrm{D} \mathrm{TMD} / \mathrm{Au}$ interfaces. We intentionally shortened the CVD growth time of $\mathrm{MoS}_{2}$ from $20 \mathrm{~min}$ to $10 \mathrm{~min}$ to make NPG internal surfaces partially covered by monolayer $\mathrm{MoS}_{2}$. Figure 8a shows the $\mathrm{Au}(001)$ surface on which the $\mathrm{Au}_{4} \mathrm{~S}_{4}$ reconstruction is only visible in the region covered by the $\mathrm{MoS}_{2}$ monolayer. With the absence of $\mathrm{MoS}_{2}$ layer in the left side of the (001) surface, the $\mathrm{Au}_{4} \mathrm{~S}_{4}$ reconstruction layer also disappears. The intensity line profiles shown in Fig. $8 \mathrm{~b}$ further verify the exact correspondence between the reconstructed $\mathrm{Au}$ (001) surface and 2D $\mathrm{MoS}_{2}$ as the $\mathrm{Au}_{4} \mathrm{~S}_{4}$ structure disappears immediately at the edge of the $\mathrm{MoS}_{2}$ monolayer. Meanwhile, the contrast variation of the $\mathrm{Au}(001)$ sublayer also disappears together with the absence of the reconstructed topmost surface. These structural features unambiguously demonstrate that the $\mathrm{Au}_{4} \mathrm{~S}_{4}$ reconstruction is stimulated and stabilized by $\mathrm{MoS}_{2}$ and the sublayer reconstruction, most likely as the buffer layer, correlates with the topmost 

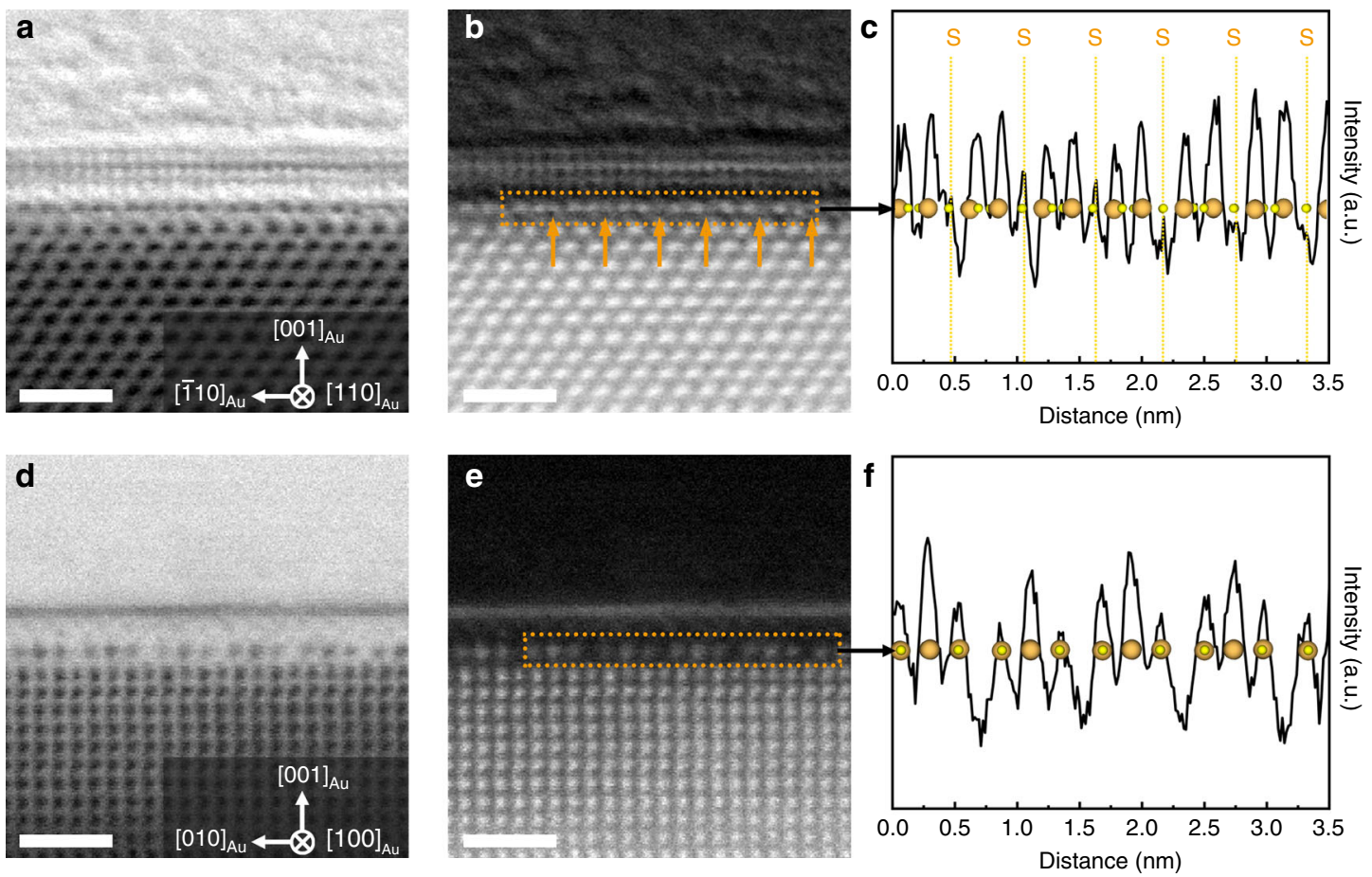

Fig. 6 Characterization of $\mathbf{S}$ atom occupation in the reconstructed Au (001) surface. a ABF-STEM image viewed from the $[110]_{\mathrm{Au}}$ direction. Scale bar, $1 \mathrm{~nm}$. b Contrast-inversed image of (a) showing the $S$ atomic columns. Scale bar, $1 \mathrm{~nm}$. $\mathbf{c}$ The corresponding intensity line profile of the reconstructed layer from dotted box in (b). d ABF-STEM image viewed from the $[100]_{A u}$ direction. Scale bar, $1 \mathrm{~nm}$. e Contrast-inversed image of (d) in which S atomic columns are invisible. Scale bar, $1 \mathrm{~nm}$. $\mathbf{f}$ The corresponding intensity line profile of the reconstructed layer from dotted box in (e). The orange and yellow spheres represent $\mathrm{Au}$ and $\mathrm{S}$ atoms, respectively.

$\mathrm{Au}_{4} \mathrm{~S}_{4}$ reconstruction. Importantly, in comparison with the unreconstructed region of the (001) surface, the $\mathrm{Au}_{4} \mathrm{~S}_{4}$ reconstruction is one atomic layer high and forms an atomic step at the site between reconstructed and unreconstructed (001) surface. It provides compelling evidence that the $\mathrm{Au}$ atoms in $\mathrm{Au}_{4} \mathrm{~S}_{4}$ monolayer most likely come from the sublayer and are "dragged" out by $\mathrm{S}$ atoms to form the two-atomic-layer interfacial phase. Similar phenomena can also be observed from other Au facets, such as $\{110\}$ and $\{113\}$ (Supplementary Fig. 8). We also carefully characterized the surface structure of the NPG sample, which is annealed in sulfur-rich environment but without the Mo source for $\mathrm{MoS}_{2}$ growth at the CVD temperature of $873 \mathrm{~K}$. Again, no reconstructed surfaces can be seen except that some sulfur particles attach on Au surfaces (Supplementary Fig. 9). We performed molecular dynamics (MD) simulations on the thermal stability of the reconstructed $\mathrm{Au}$ (001) surface with or without $\mathrm{MoS}_{2}$ coverage (for details, see the Methods section). The reconstructed surface without $\mathrm{MoS}_{2}$ coverage disappears shortly after $0.2 \mathrm{ps}$ by decomposing as elemental $\mathrm{S}$ atoms and $\mathrm{Au}$ (Fig. 8c). In contrast, when covered by $\mathrm{MoS}_{2}$, the reconstructed $\mathrm{Au}_{4} \mathrm{~S}_{4}$ structure can retain well at $900 \mathrm{~K}$ in the maximum simulation time period of $10 \mathrm{ps}$ in this study (Fig. 8d). The enhanced thermal stability of the interfacial phase is apparently related to the vdW interaction with the top $\mathrm{MoS}_{2}$ monolayers although further investigation is required ${ }^{50}$.

We also investigated the effects of excess sulfur, CVD temperatures and Mo sources on the interfacial reconstructions. After largely decreasing the loading amount of sulfur powders from $1 \mathrm{~g}$ to $200 \mathrm{mg}$ for CVD growth, unreconstructed interfaces between $\mathrm{MoS}_{2}$ and Au can be observed (Supplementary Fig. 10), suggesting that the S-rich environment is necessary for the formation of the interfacial $\mathrm{Au}_{4} \mathrm{~S}_{4}$ phase. In contrast, when we changed the Mo source from $\mathrm{MoO}_{3}$ to $\mathrm{MoCl}_{5}$ and reduced the CVD temperature from 923 to $723 \mathrm{~K}$, the interfacial reconstruction is not affected and always occurs accompanying with the formation of $\mathrm{MoS}_{2}$ (Supplementary Fig. 11).

\section{Discussion}

We noticed that the sulfur-induced gold surface reconstruction, in turn, alters the structure of $2 \mathrm{D}$ TMDs. It was found that the reconstructed Au surface leads to the inverse symmetry breaking of the S-Mo-S in top $1 \mathrm{H} \mathrm{MoS}_{2}$. The distances between Mo layer and two $S$ layers, which should be the same as $d_{0} \sim 1.62 \AA$, change to two different values $\left(d_{1}\right.$ and $\left.d_{2}\right)$. The one that is close to the reconstructed interface is $2.05 \AA \pm 0.08 \AA\left(d_{2}\right.$ in Fig. 9h), which is obviously larger than $d_{1}$ that remains to be close to $d_{0}\left(d_{1}, \sim 1.65 \AA\right)$ (Fig. 9a-d). In addition to the asymmetric S-Mo-S structure of $1 \mathrm{H}$ $\mathrm{MoS}_{2}$, the distance between the reconstructed Au surface and the Mo layers also becomes smaller ( $4.65 \AA \pm 0.27 \AA, h_{1}$ in Fig. 9h) in comparison with that of the unreconstructed interface $\left(h_{0}\right.$, $\sim 5.30 \AA)^{13}$. Furthermore, the interlayer spacing between the first two $\mathrm{MoS}_{2}$ layers was significantly expanded when multi-layer $\mathrm{MoS}_{2}$ was grown on NPG by increasing the deposition time (Fig. 9e). The magnified HAADF-STEM image (Fig. 9f) clearly shows the enlarged interlayer spacing of $11.42 \AA \pm 1.51 \AA\left(c_{2}\right.$ in Fig. 9h), which is almost twice larger than the normal one $(6.15 \AA)$. Although the vertical distance between the bottom $\mathrm{S}$ layer and the topmost $\mathrm{Au}_{4} \mathrm{~S}_{4}$ is $\sim 2.56 \AA$, the minimum interatomic distance between a sulfur atom in the bottom $\mathrm{S}$ layer of $\mathrm{MoS}_{2}$ and a gold atom in the topmost $\mathrm{Au}_{4} \mathrm{~S}_{4}$ layer is $3.04 \AA$ (Supplementary Fig. 12b), which is much larger than the covalent $\mathrm{Au}-\mathrm{S}$ bonding lengths $(\sim 2.42 \AA)^{8}$. Therefore, the interaction between monolayer $\mathrm{MoS}_{2}$ and $\mathrm{Au}_{4} \mathrm{~S}_{4}$ reconstructed surface should still be the vdW type. This assumption is supported by DFT calculations. In the isosurface of partial charge density, there is no charge redistribution and superposition at the interface between $\mathrm{MoS}_{2}$ and reconstructed $\mathrm{Au}_{4} \mathrm{~S}_{4}$ (Supplementary Fig. 12c). 
a
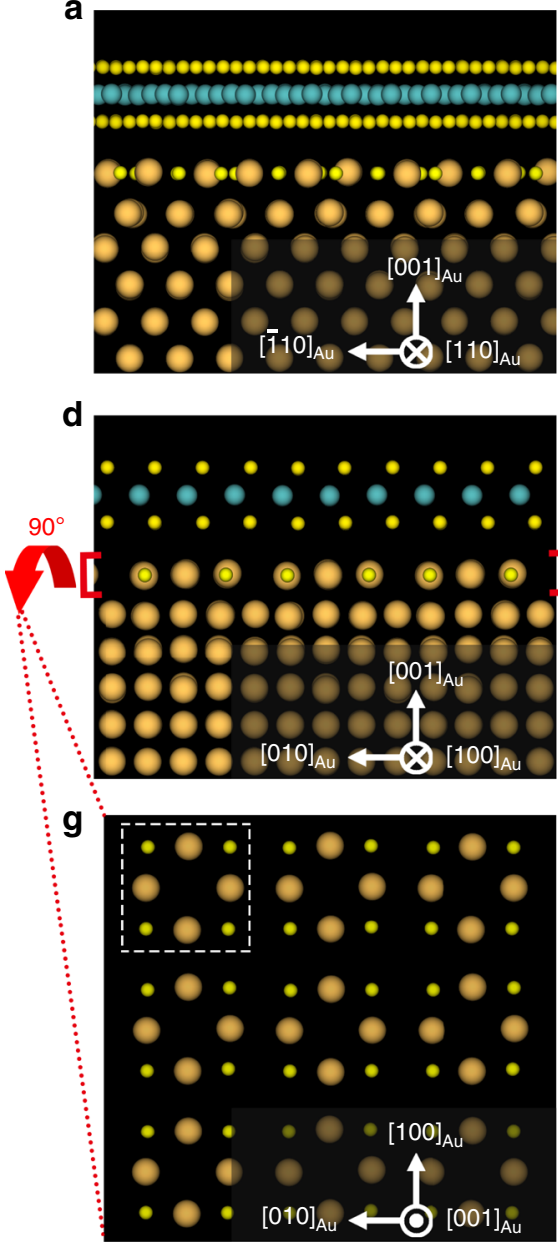
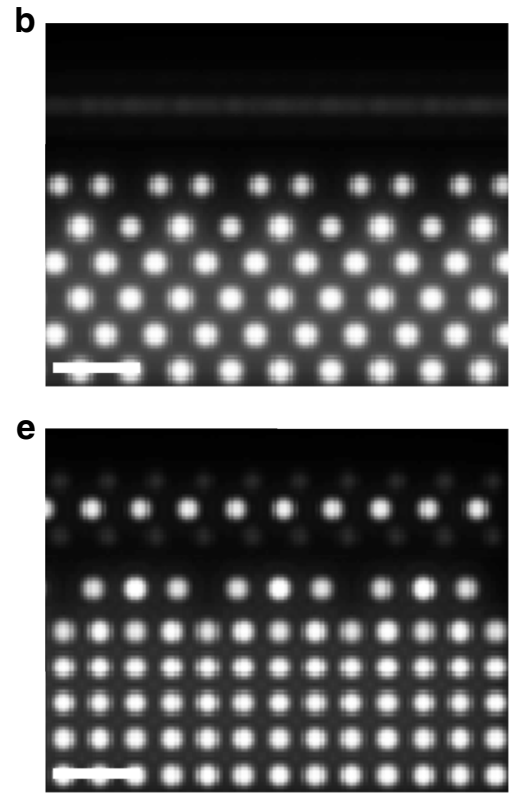

h

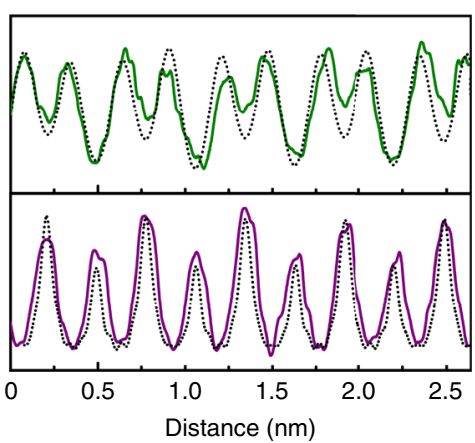

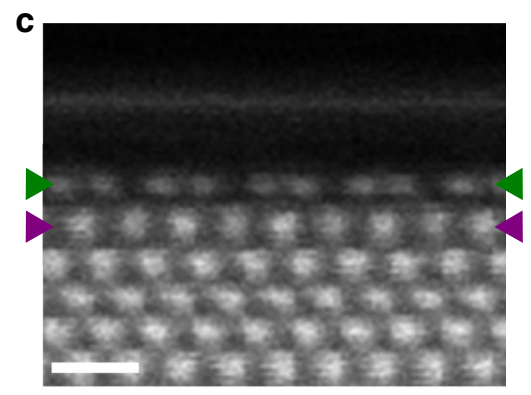
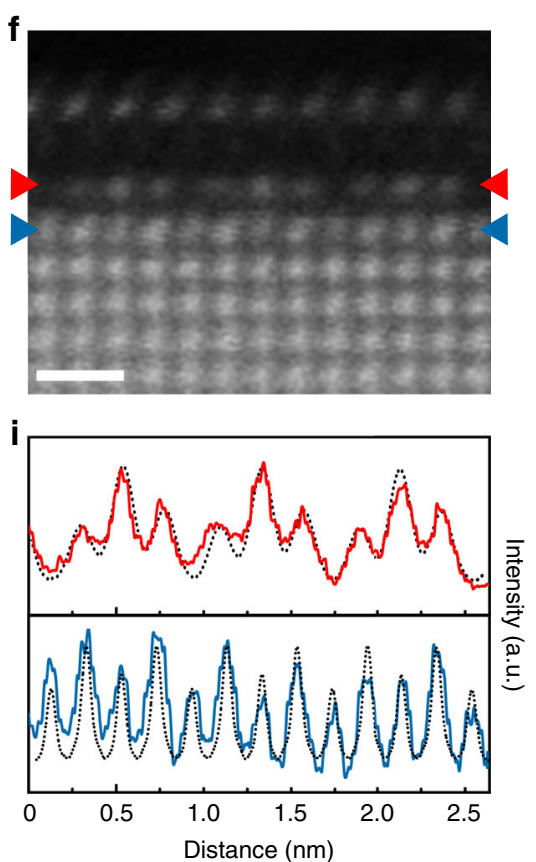

Fig. 7 Atomic configuration of the reconstructed interface between $\mathbf{M o S}_{\mathbf{2}}$ and $\mathbf{A u}(\mathbf{0 0 1}) . \mathbf{a}, \mathbf{d}$ The DFT-optimized structure model viewed from the $[110]_{\mathrm{Au}}$ (a) and [010] $\mathrm{Au}(\mathbf{d})$ directions, respectively. b, e Simulated HAADF-STEM images from the DFT-optimized structure model viewed from the [110] ${ }_{\mathrm{Au}}$ (b) and [010] $\mathrm{Au}(\mathbf{e})$ directions. Scale bar, $0.5 \mathrm{~nm}$. c, f Experimental HAADF-STEM images showing good match with the simulated ones viewed from the $[110]_{\mathrm{Au}}(\mathbf{c})$ and [010] $\mathrm{Au}$ (f) directions. Scale bar, $0.5 \mathrm{~nm}$. $\mathbf{g}$ The reconstructed Au (001) surface viewed from the [001] Au direction (i.e., top view). The $\mathrm{MoS}_{2}$ monolayer and Au matrix are omitted for clarity. The dotted box shows the unit cell of an $\mathrm{Au}_{4} \mathrm{~S}_{4}$ ring. $\mathbf{h}$, $\mathbf{i}$ Line profiles showing the intensity variation of atomic columns viewed from the $[110]_{\mathrm{Au}}(\mathbf{h})$ and $[010]_{\mathrm{Au}}(\mathbf{i})$ directions. The green and purple lines $(\mathbf{h})$ are detected along the $\mathrm{Au}_{4} \mathrm{~S}_{4}$ monolayer and Au sublayer following the corresponding arrows in (c). The red and blue lines (i) are detected along the $\mathrm{Au}_{4} \mathrm{~S}_{4} \mathrm{monolayer}_{\mathrm{and}} \mathrm{Au}$ sublayer following the corresponding arrows in (f). The dashed lines are detected from the same areas in corresponding simulated images. The orange, green and yellow spheres represent $\mathrm{Au}, \mathrm{Mo}$, and $\mathrm{S}$ atoms, respectively.

Since structural changes often result in new functionalities of materials, the interfacial reconstruction between $\mathrm{MoS}_{2}$ and $\mathrm{Au}$ may open a way to tailor the properties of $2 \mathrm{D}$ TMDs and their devices. Our preliminary DFT study (Fig. 10) suggests that the formation of $\mathrm{Au}_{4} \mathrm{~S}_{4}$ interfacial phase leads to the transition of the original n-type vdW contact between $\mathrm{MoS}_{2}$ monolayer and $\mathrm{Au}(001)$ electrode to a p-type contact with the reduced Schottky barrier height from $0.89 \mathrm{eV}\left(\Phi_{\mathrm{SB}, \mathrm{N}}=E_{\mathrm{CB}}-E_{\mathrm{f}}\right)$ to $0.45 \mathrm{eV}\left(\Phi_{\mathrm{SB}}\right.$, $\left.\mathrm{P}=E_{\mathrm{VB}}-E_{\mathrm{f}}\right)$. This transition may be utilized to realize the $\mathrm{n} / \mathrm{p}$-type conversion in 2D TMD devices and the lower energy barrier may benefit the hole mobility in p-type transistors ${ }^{51,52}$. While, the DFT calculations show that the vertically distorted $\mathrm{MoS}_{2}$ monolayer on the reconstructed $\mathrm{Au}_{4} \mathrm{~S}_{4}$ is not energetically stable and becomes to be more symmetric during relaxation. The divergence between our experimental observations and DFT calculations could be caused by the complexity of the real interfacial structure. Although the vertical distortion alone can obviously affect the bandgaps of $\mathrm{MoS}_{2}$ (Supplementary Fig. 13), it may not significantly alter the vdW contact between $\mathrm{MoS}_{2}$ monolayer and $\mathrm{Au}$ (001) surface in which the reconstructed $\mathrm{Au}_{4} \mathrm{~S}_{4}$ interfacial phase plays a more important role in the transition from n-type to p-type vdW contacts.

In conclusion, we observed the interfacial reconstruction at $\mathrm{Au}$ (001)/monolayer TMDs junctions. The reconstructed $\mathrm{Au}_{4} \mathrm{~S}_{4}$ structure co-exists with and is stabilized by the top TMD monolayers with an enhanced $\mathrm{vdW}$ interaction. The unique $\mathrm{vdW}$ contact between TMDs and Au by interfacial reconstruction may pave a new way to fabricate perfect vdW interface with tunable the Schottky barrier height and contact types and to develop new functionalities of TMDs-metal heterostructures.

\section{Methods}

Preparation of NPG substrates. The NPG substrates were prepared by chemically dealloying $\mathrm{Ag}_{65} \mathrm{Au}_{35}$ (atomic percentage) films with a thickness of $100 \mathrm{~nm}$ in a 70 vol\% $\mathrm{HNO}_{3}$ solution (Aldrich) for $3 \mathrm{~h}$ at room temperature ${ }^{33}$, by which silver was selectively dissolved from the alloy and left behind an NPG membrane. After removing the residual chemicals by rinsing, the NPG films were placed on a silicon wafer with $200 \mathrm{~nm}$ thick $\mathrm{SiO}_{2}$ as the $3 \mathrm{D}$ porous substrates for CVD growth of $\mathrm{MoS}_{2}$ or $\mathrm{WS}_{2}$. 

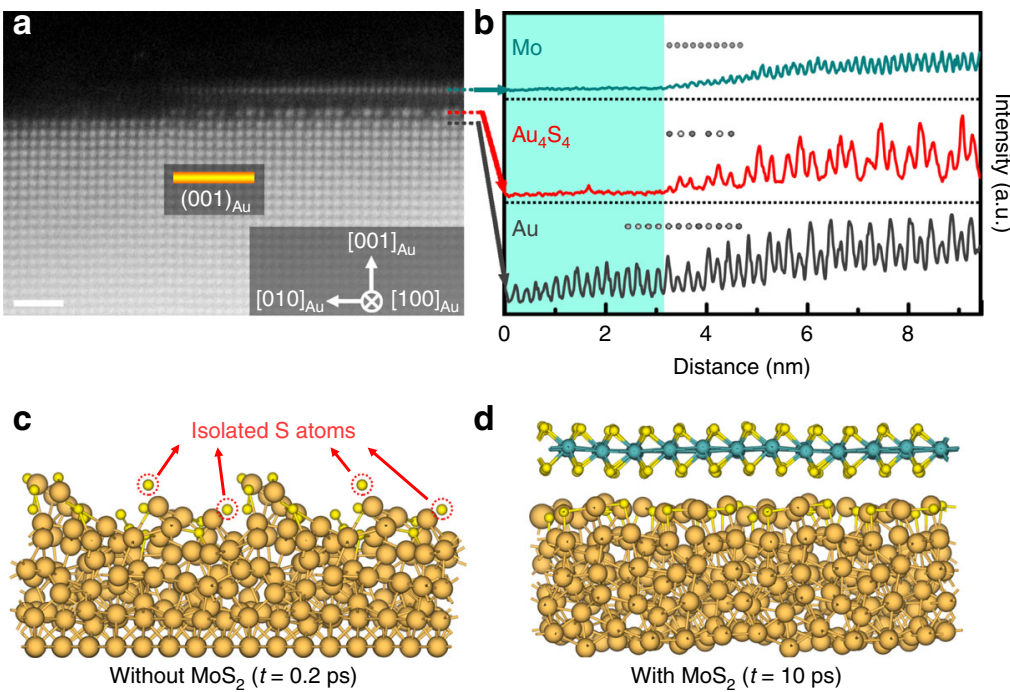

Fig. 8 The co-existence relationship between $\mathbf{M o S}_{\mathbf{2}}$ and reconstructed $\mathbf{A u}_{\mathbf{4}} \mathbf{S}_{\mathbf{4}}$ structure. a HAADF-STEM image of a partially covered Au (001) surface

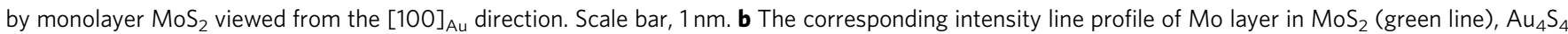
interface layer (red line) and Au sublayer (gray line). c, d Stability of the $\mathrm{Au}_{4} \mathrm{~S}_{4}$ phase in MD simulations without (c) or with (d) a monolayer MoS 2 top. The orange, green and yellow spheres represent $\mathrm{Au}, \mathrm{Mo}$, and $\mathrm{S}$ atoms, respectively.
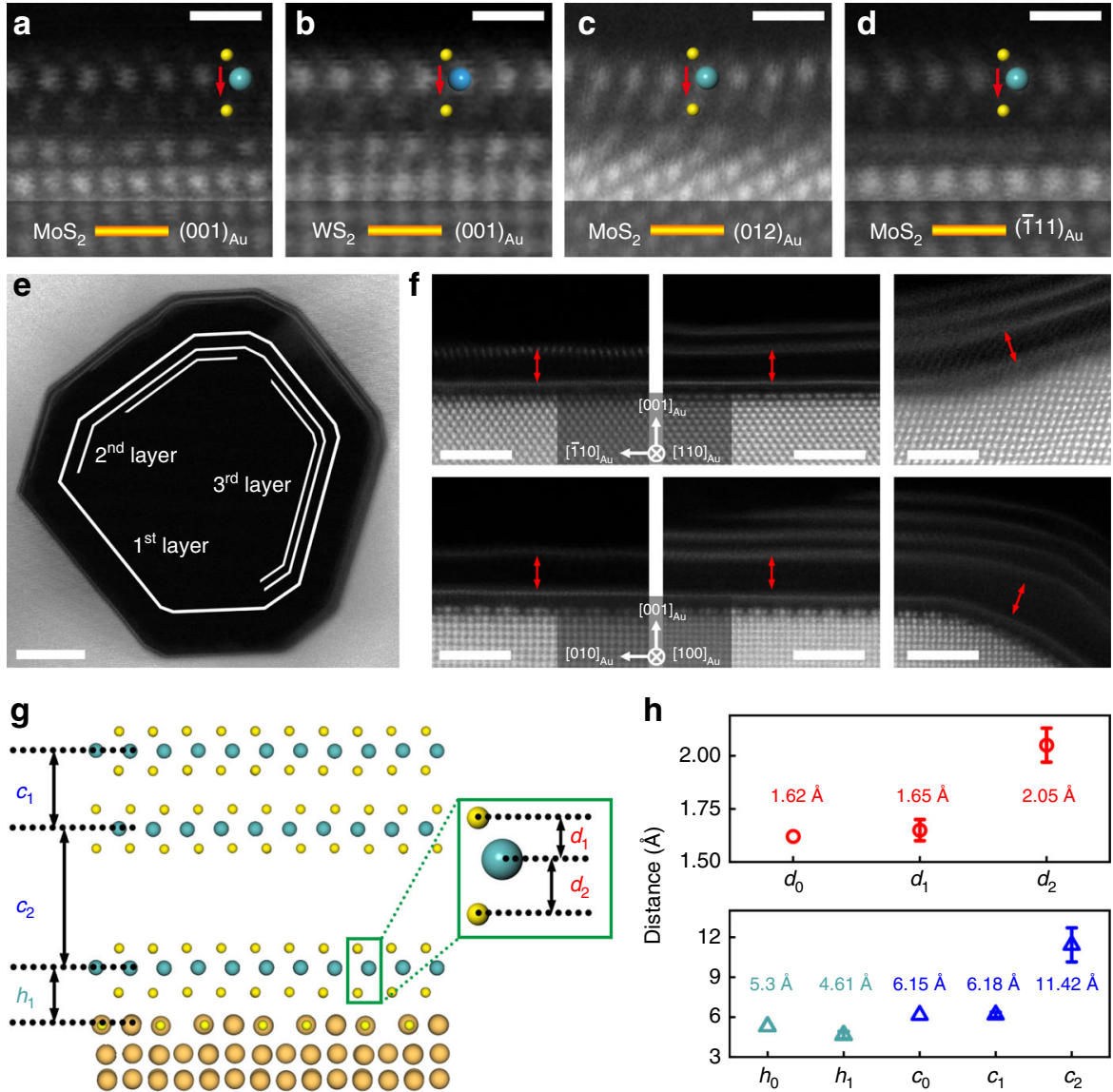

Fig. 9 Structure of monolayer and multi-layer $\mathbf{M o S}_{\mathbf{2}}$ on reconstructed Au (001) surfaces. a-d HAADF-STEM imaging showing the cross-sectional structures of $\mathrm{MoS}_{2}$ and $\mathrm{WS}_{2}$ monolayers on the reconstructed Au (001) surfaces. Scale bar, $0.5 \mathrm{~nm}$. e Multi-layer MoS ${ }_{2}$ on the internal surface of NPG. Scale bar, $5 \mathrm{~nm}$. f Atomic-resolution HAADF-STEM images of multi-layer $\mathrm{MoS}_{2}$ grown on Au surfaces viewed from the $[100]_{\mathrm{Au}}$ and $[110]_{\mathrm{Au}}$ directions. Scale bar, $2 \mathrm{~nm}$. $\mathbf{g}$ Structure model of the $\mathrm{MoS}_{2}$ on the reconstructed Au (001) surface. The $d$ is defined as the distance between Mo atom layer and $\mathrm{S}$ atom layer, $c$ is the interlayer spacing between two $\mathrm{MoS}_{2}$ layers and $h$ is the distance between topmost Au atom layer and the nearest $S$ atom layer in the top $\mathrm{MoS}_{2}$. $\mathbf{h}$ The comparison of $d_{1} h$, and $c$ with the values of $d_{0}, h_{0}$ (ref. ${ }^{13}$ ), and $c_{0}$ in pristine $\mathrm{MoS}_{2}$. The error bars represent the standard deviation of each distance. Source data are provided as a Source Data file. The orange, green, blue and yellow spheres represent Au, Mo, W, and S atoms, respectively. 
a
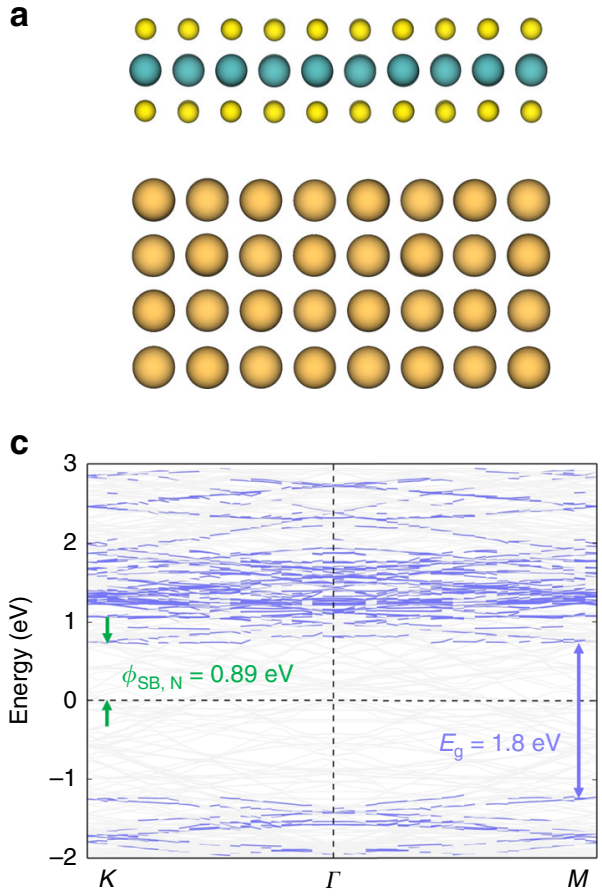

b

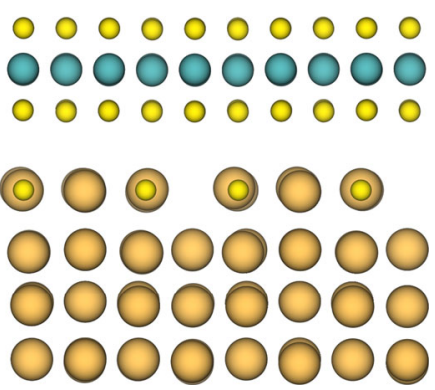

d

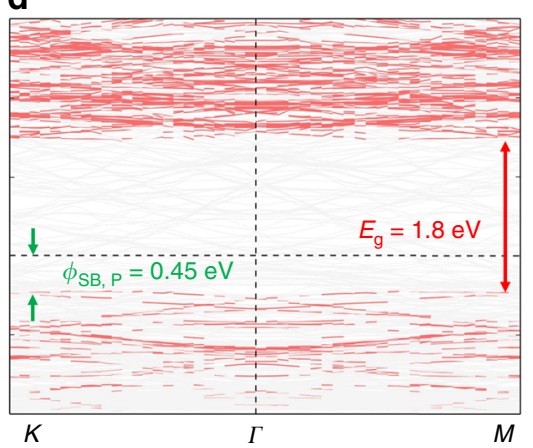

Fig. 10 The band structures of $\mathbf{M o S}_{\mathbf{2}}-\mathbf{A u}(\mathbf{0 0 1})$ interface without and with $\mathbf{A u}_{\mathbf{4}} \mathbf{S}_{\mathbf{4}}$ reconstruction. $\mathbf{a}, \mathbf{c}$ The side view of the model of unreconstructed $\mathrm{MoS}_{2}-\mathrm{Au}(001)$ interface (a) and the corresponding band structure (c). b, d The side view of the model of reconstructed $\mathrm{MoS}_{2}-\mathrm{Au}_{4} \mathrm{~S}_{4}$ interface $(\mathbf{b})$ and the corresponding band structure (d). The orange, green and yellow spheres represent $A u, M o$, and $S$ atoms, respectively. The zero of energy is set at the Fermi level. $E_{g}$ denotes the band gap of monolayer $\mathrm{MoS}_{2}$, and $\Phi_{\mathrm{SB}, \mathrm{N}}\left(\Phi_{\mathrm{SB}, \mathrm{P}}\right)$ denotes n-type (p-type) SBH for electrons (holes).

CVD growth of TMDs on NPG. The $\mathrm{MoS}_{2}$ or $\mathrm{WS}_{2}$ monolayer and multilayer films were grown on NPG using a three-temperature-zone CVD system. As shown in Supplementary Fig. 1, $\sim \mathrm{g}$ sulfur (Aldrich, purity $99.9 \%$ ), $\sim 0.02 \mathrm{~g} \mathrm{MoO}_{3}$ powders (Aldrich, purity $99.9 \%$ ) and the substrate (100 nm thickness NPG on oxidized silicon) were placed from upstream to downstream at the three zones with different temperatures, respectively. Ultra-pure argon was used as the carrier gas and the pressure inside the tube was controlled by adjusting the Ar flow rate. During the reaction process, the $\mathrm{Ar}$ flow rate was $600 \mathrm{sccm}$, and the temperatures of three zones were $120^{\circ} \mathrm{C}, 550^{\circ} \mathrm{C}$ and $650^{\circ} \mathrm{C}$, respectively. After the reaction time of $20 \mathrm{~min}, \mathrm{MoS}_{2}$ monolayers completely covered the internal surfaces of NPG. Multilayer $\mathrm{MoS}_{2}$ films were obtained by extending the reaction time to $30 \mathrm{~min}$. As shown in Supplementary Fig. 1c, the synthesis of $\mathrm{WS}_{2}$ films was similar to that of $\mathrm{MoS}_{2} . \sim 0.02 \mathrm{~g} \mathrm{WCl}_{6}$ powders (Aldrich, purity $99.9 \%$ ) and $\sim 1 \mathrm{~g}$ sulfur (Aldrich, purity $99.9 \%$ ) were placed from upstream to downstream respectively as precursor sources, and the heating temperature were $55^{\circ} \mathrm{C}$ and $120^{\circ} \mathrm{C}$ during the CVD process.

STEM Characterization. The microstructures and atomic structures of $\mathrm{MoS}_{2}$ and $\mathrm{WS}_{2}$ on NPG were characterized by a transmission electron microscope (JEOL ARM 200F) equipped with a cold emission gun and an aberration corrector for the probe-forming lens system. The collection angle of the HAADF detector ranges from 81 to $228 \mathrm{mrad}$, and the collection angle of the ABF detector ranges from 6 to $25 \mathrm{mrad}$. The filtered STEM-HAADF images with temperature color in Figs. 1 and 3 were deconvoluted with HREM DeConvHAADF software (HREM Research Inc. $)^{53}$. The rest images used in the paper are all original ones.

EELS Characterization. The EELS analyses were performed with the Gatan GIF Quantum system on STEM mode. The EELS elemental mapping was simultaneously recorded with HAADF-STEM. The pixel size is $0.32 \AA \times 0.32 \AA$ and a short dwell time of $0.01 \mathrm{~s} /$ pixel was used in order to avoid the spatial drift and irradiation damage. $S L_{2,3}$ edges presented in Fig. 1e are the integrated signals from all the pixels in 3 rows for each defined layer (Supplementary Fig. 14). The width of 3 rows $(0.96 \AA)$ is smaller than the atomic radius (S, $1.09 \AA$; Mo, $1.36 \AA$; $A u, 1.44 \AA$ ). There is a gap spacing between two adjacent layers with more than 4 rows ( $1.28 \AA$ ) to avoid possible interference from scanning drifts. In this way, we got the original EELS spectra of these six layers (Supplementary Fig. 15). To better show the characteristic EELS edges and peaks, we smoothed these spectra with low-pass numerical filtering. The background of sulfur core-loss edges was subtracted using a power law fitting. All the smoothing and subtracting processes are performed using the Digital Micrograph software package (Gatan Inc.).
STEM image simulation. The simulations of HAADF-STEM images in Fig. 7 were conducted by $x$ HREM software with STEM Extension (HREM Research Inc.) ${ }^{54}$, and the absorption of thermal diffuse scattering was taken into account for each element. The defocus value of $\sim 5 \mathrm{~nm}$ was adopted and the specimen thickness of $\sim 5 \mathrm{~nm}$ was used. The $200 \mathrm{kV}$ probe with a probe-forming aperture of $22 \mathrm{mrad}$ was postulated to be aberration-free. The collection angle of the HAADF detector is set from 81 to $228 \mathrm{mrad}$.

DFT calculations. Density functional theory based First-principles calculations were carried out using FHI-aims code ${ }^{55,56}$, which is an all electron, full potential electronic structure code by using a numeric, atom-centered basis set. The electronic exchange and correlation were treated with the generalized gradient approximation $(\mathrm{GGA})$ of Perdew-Burke-Ernzerhof $(\mathrm{PBE})^{57}$. All the numeric settings were chosen as an energy convergence less than $10^{-3} \mathrm{eV}$ is achieved. The atomic positions were allowed to fully relax using the Broyden-Fletcher-Goldfarb-Shanno (BFGS) algorithm. Using DFT implemented CP2K package ${ }^{58,59}$, ab initio molecular dynamics simulations for the reconstructed and unreconstructed interface were performed in the canonical ensemble by using the Nose-Hoover thermostat and a time step of $1 \mathrm{fs}$ at a finite MD temperature of $900 \mathrm{~K}$. The exchange correlation potential was described by the GGA with the spin-polarized functional of $\mathrm{PBE}^{57}$. Wavefunctions were expanded in triple- $\zeta$ Gaussian basis sets with an auxiliary plane-wave basis and a cutoff energy of $300 \mathrm{Ry}$. Core electrons were modeled by scalar relativistic normconserving pseudopotentials ${ }^{60,61}$.

\section{Data availability}

The source data underlying Fig. $9 \mathrm{~h}$ are provided as a Source Data file. Other data that support the findings of this study are available from the corresponding authors upon request.

Received: 27 September 2019; Accepted: 29 January 2020; Published online: 21 February 2020

\section{References}

1. Wang, Q. H., Kalantar-Zadeh, K., Kis, A., Coleman, J. N. \& Strano, M. S. Electronics and optoelectronics of two-dimensional transition metal dichalcogenides. Nat. Nanotechnol. 7, 699-712 (2012).

2. Splendiani, A. et al. Emerging photoluminescence in monolayer $\mathrm{MoS}_{2}$. Nano Lett. 10, 1271-1275 (2010). 
3. Ding, Q., Song, B., Xu, P. \& Jin, S. Efficient electrocatalytic and photoelectrochemical hydrogen generation using $\mathrm{MoS}_{2}$ and related compounds. Chem 1, 699-726 (2016).

4. Rahaman, M. et al. Highly localized strain in a $\mathrm{MoS}_{2} / \mathrm{Au}$ heterostructure revealed by tip-enhanced Raman spectroscopy. Nano Lett. 17, 6027-6033 (2017).

5. Li, Y. et al. Morphological engineering of winged $\mathrm{Au} @ \mathrm{MoS}_{2}$ heterostructures for electrocatalytic hydrogen evolution. Nano Lett. 18, 7104-7110 (2018).

6. Schulman, D. S., Arnold, A. J. \& Das, S. Contact engineering for 2D materials and devices. Chem. Soc. Rev. 47, 3037-3058 (2018)

7. Liu, Y., Huang, Y. \& Duan, X. Van der Waals integration before and beyond two-dimensional materials. Nature 567, 323-333 (2019).

8. Popov, I., Seifert, G. \& Tománek, D. Designing electrical contacts to $\mathrm{MoS}_{2}$ monolayers: a computational study. Phys. Rev. Lett. 108, 156802 (2012).

9. Kang, J., Liu, W., Sarkar, D., Jena, D. \& Banerjee, K. Computational study of metal contacts to monolayer transition-metal dichalcogenide semiconductors. Phys. Rev. X 4, 031005 (2014).

10. Gong, C., Colombo, L., Wallace, R. M. \& Cho, K. The unusual mechanism of partial fermi level pinning at metal- $\mathrm{MoS}_{2}$ interfaces. Nano Lett. 14, 1714-1720 (2014).

11. Allain, A., Kang, J., Banerjee, K. \& Kis, A. Electrical contacts to twodimensional semiconductors. Nat. Mater. 14, 1195-1205 (2015).

12. Kim, C. et al. Fermi level pinning at electrical metal contacts of monolayer molybdenum dichalcogenides. ACS Nano 11, 1588-1596 (2017).

13. Liu, Y. et al. Approaching the Schottky-Mott limit in van der Waals metal-semiconductor junctions. Nature 557, 696-700 (2018).

14. Wu, R. J. et al. Visualizing the metal- $\mathrm{MoS}_{2}$ contacts in two-dimensional fieldeffect transistors with atomic resolution. Phys. Rev. Mater. 3, 111001 (2019).

15. Chee, S.-S. et al. Lowering the schottky barrier height by graphene/Ag electrodes for high-mobility $\mathrm{MoS}_{2}$ field-effect transistors. Adv. Mater. 31, 1804422 (2019).

16. Murthy, A. A. et al. Intrinsic transport in $2 \mathrm{D}$ heterostructures mediated through h-BN tunneling contacts. Nano Lett. 18, 2990-2998 (2018).

17. Wang, Y. et al. Van der Waals contacts between three-dimensional metals and two-dimensional semiconductors. Nature 568, 70-74 (2019).

18. Cai, Z., Liu, B., Zou, X. \& Cheng, H.-M. Chemical vapor deposition growth and applications of two-dimensional materials and their heterostructures. Chem. Rev. 118, 6091-6133 (2018).

19. $\mathrm{Xu}, \mathrm{X}$. et al. Ultrafast epitaxial growth of metre-sized single-crystal graphene on industrial $\mathrm{Cu}$ foil. Sci. Bull. 62, 1074-1080 (2017).

20. Wang, L. et al. Epitaxial growth of a 100-square-centimetre single-crystal hexagonal boron nitride monolayer on copper. Nature 570, 91-95 (2019)

21. Ji, Q., Zhang, Y., Zhang, Y. \& Liu, Z. Chemical vapour deposition of groupVIB metal dichalcogenide monolayers: engineered substrates from amorphous to single crystalline. Chem. Soc. Rev. 44, 2587-2602 (2015).

22. Tan, Y. et al. Monolayer $\mathrm{MoS}_{2}$ films supported by 3D nanoporous metals for high-efficiency electrocatalytic hydrogen production. Adv. Mater. 26, 8023-8028 (2014).

23. Shi, J. et al. Controllable growth and transfer of monolayer $\mathrm{MoS}_{2}$ on Au foils and its potential application in hydrogen evolution reaction. ACS Nano 8, 10196-10204 (2014).

24. Shi, J. et al. Substrate facet effect on the growth of monolayer $\mathrm{MoS}_{2}$ on $\mathrm{Au}$ foils. ACS Nano 9, 4017-4025 (2015).

25. Gao, Y. et al. Large-area synthesis of high-quality and uniform monolayer $\mathrm{WS}_{2}$ on reusable Au foils. Nat. Commun. 6, 8569 (2015).

26. Häkkinen, H. The gold-sulfur interface at the nanoscale. Nat. Chem. 4, 443-455 (2012).

27. Walen, H. et al. Identification of $\mathrm{Au}-\mathrm{S}$ complexes on $\mathrm{Au}(100)$. Phys. Chem Chem. Phys. 18, 4891-4901 (2016).

28. Zhang, Y. et al. 3D bicontinuous nanoporous plasmonic heterostructure for enhanced hydrogen evolution reaction under visible light. Nano Energy 58, 552-559 (2019).

29. Yin, X. et al. Tunable inverted gap in monolayer quasi-metallic $\mathrm{MoS}_{2}$ induced by strong charge-lattice coupling. Nat. Commun. 8, 486 (2017).

30. Liu, Z. et al. Synthesis of $\operatorname{MoX}_{2}(\mathrm{X}=\mathrm{Se}$ or $\mathrm{S})$ monolayers with highconcentration $1 \mathrm{~T}^{\prime}$ phase on $4 \mathrm{H} / \mathrm{fcc}-\mathrm{Au}$ nanorods for hydrogen evolution. Nano Res. 12, 1301-1305 (2019).

31. Sørensen, S. G., Füchtbauer, H. G., Tuxen, A. K., Walton, A. S. \& Lauritsen, J. V. Structure and electronic properties of in situ synthesized single-layer $\mathrm{MoS}_{2}$ on a gold surface. ACS Nano 8, 6788-6796 (2014).

32. Zhou, X. et al. Periodic modulation of the doping level in striped $\mathrm{MoS}_{2}$ superstructures. ACS Nano 10, 3461-3468 (2016).

33. Erlebacher, J., Aziz, M. J., Karma, A., Dimitrov, N. \& Sieradzki, K. Evolution of nanoporosity in dealloying. Nature 410, 450-453 (2001).

34. Wang, Z. et al. Chemical vapor deposition of monolayer $\mathrm{Mo}_{1-\mathrm{x}} \mathrm{W}_{\mathrm{x}} \mathrm{S}_{2}$ crystals with tunable band gaps. Sci. Rep. 6, 21536 (2016).

35. Wang, Z. et al. Engineering the internal surfaces of three-dimensional nanoporous catalysts by surfactant-modified dealloying. Nat. Commun. 8, 1066 (2017).

36. Li, H. et al. From bulk to monolayer $\mathrm{MoS}_{2}$ : evolution of Raman scattering. Adv. Funct. Mater. 22, 1385-1390 (2012).
37. Zhou, W. et al. Intrinsic structural defects in monolayer molybdenum disulfide. Nano Lett. 13, 2615-2622 (2013).

38. Hong, J. et al. Exploring atomic defects in molybdenum disulphide monolayers. Nat. Commun. 6, 6293 (2015).

39. Han, S. A. et al. Point-defect-passivated $\mathrm{MoS}_{2}$ nanosheet-based high performance piezoelectric nanogenerator. Adv. Mater. 30, 1800342 (2018)

40. Fujita, T. et al. Atomic origins of the high catalytic activity of nanoporous gold. Nat. Mater. 11, 775-780 (2012).

41. Liu, P. et al. Visualizing under-coordinated surface atoms on 3D nanoporous gold catalysts. Adv. Mater. 28, 1753-1759 (2016).

42. Dadsetani, M., Nejatipour, H. \& Nouri, T. First-principles study of the sulfur $\mathrm{K}$ and L2,3 edges of transition metal disulfide monolayers, $\mathrm{MS}_{2}(\mathrm{M}=\mathrm{Mo}, \mathrm{W}$ and Re). Phys. E Low.-Dimens. Syst. Nanostruct. 73, 198-206 (2015).

43. Pennycook, S. J. \& Boatner, L. Chemically sensitive structure-imaging with a scanning transmission electron microscope. Nature 336, 565 (1988).

44. Hartel, P., Rose, H. \& Dinges, C. Conditions and reasons for incoherent imaging in STEM. Ultramicroscopy 63, 93-114 (1996).

45. Jacobsen, J. L. On the universality of fully packed loop models. J. Phys. Math. Gen. 32, 5445-5453 (1999).

46. Kargarian, M. \& Fiete, G. A. Topological phases and phase transitions on the square-octagon lattice. Phys. Rev. B 82, 085106 (2010).

47. Bao, A., Tao, H.-S., Liu, H.-D., Zhang, X. \& Liu, W.-M. Quantum magnetic phase transition in square-octagon lattice. Sci. Rep. 4, 6918 (2015).

48. Jiang, D., Tiago, M. L., Luo, W. \& Dai, S. The "Staple" Motif: a key to stability of thiolate-protected gold nanoclusters. J. Am. Chem. Soc. 130, 2777-2779 (2008)

49. Kenzler, S., Schrenk, C. \& Schnepf, A. $\mathrm{Au}_{108} \mathrm{~S}_{24}\left(\mathrm{PPh}_{3}\right)_{16}$ : Bestätigung des allgemeinen Konzeptes metalloider Cluster durch einen hochsymmetrischen nanoskaligen Goldcluster. Angew. Chem. 129, 402-406 (2017).

50. Hwang, J.-Y., Kim, Y.-M., Lee, K. H., Ohta, H. \& Kim, S. W. Te monolayerdriven spontaneous van der Waals epitaxy of two-dimensional pnictogen chalcogenide film on sapphire. Nano Lett. 17, 6140-6145 (2017)

51. Chuang, S. et al. $\mathrm{MoS}_{2}$ P-type transistors and diodes enabled by high work function $\mathrm{MoO}_{x}$ contacts. Nano Lett. 14, 1337-1342 (2014).

52. Farmanbar, M. \& Brocks, G. Ohmic Contacts to 2D Semiconductors through van der Waals Bonding. Adv. Electron. Mater. 2, 1500405 (2016).

53. Ishizuka, K. \& Abe, E. Improvement of spatial resolution of STEM-HAADF image by maximum-entropy and Richardson-Lucy deconvolution. in Proceedings of the 13th European Microscopy Congress (13th EMC) Instrumentation and Methol, vol. 1, 117 (2004).

54. Ishizuka, K. A practical approach for STEM image simulation based on the FFT multislice method. Ultramicroscopy 90, 71-83 (2002).

55. Hohenberg, P. \& Kohn, W. Inhomogeneous electron gas. Phys. Rev. 136, B864-B871 (1964).

56. Blum, V. et al. Ab initio molecular simulations with numeric atom-centered orbitals. Comput. Phys. Commun. 180, 2175-2196 (2009)

57. Perdew, J. P., Burke, K. \& Ernzerhof, M. Generalized gradient approximation made simple. Phys. Rev. Lett. 77, 3865-3868 (1996).

58. Lippert, G., Hutter, J. \& Parrinello, M. The Gaussian and augmented-plane-wave density functional method for ab initio molecular dynamics simulations. Theor Chem. Acc. Theory Comput. Model. Theor. Chim. Acta 103, 124-140 (1999).

59. VandeVondele, J. et al. Quickstep: Fast and accurate density functional calculations using a mixed Gaussian and plane waves approach. Comput. Phys. Commun. 167, 103-128 (2005).

60. Goedecker, S., Teter, M. \& Hutter, J. Separable dual-space Gaussian pseudopotentials. Phys. Rev. B 54, 1703-1710 (1996).

61. Krack, M. Pseudopotentials for $\mathrm{H}$ to $\mathrm{Kr}$ optimized for gradient-corrected exchange-correlation functionals. Theor. Chem. Acc. 114, 145-152 (2005).

\section{Acknowledgements}

This work was sponsored by MOST 973 of China (Grant No. 2015CB856800), Nationa Natural Science Foundation of China (Grant Nos. 51821001, 11704245, 21773287 and 11974195) and Natural Science Foundation of Shanghai (Grant No. 19ZR1475200). The computations were performed at the Supercomputing Center in Guangzhou (NSCC-GZ) P.L. was supported by the Program for Professor of Special Appointment (Eastern Scholar) at Shanghai Institutions of Higher Learning. M.C. was supported by the Whiting School of Engineering, Johns Hopkins University.

\section{Author contributions}

M.C. conceived and supervised this study. R.L. designed and performed the experiments and conducted the sample fabrication, and TEM and Raman characterization. P.L. supervised TEM study and contributed to DFT and MD simulations. W.X. and Y.G. performed the DFT and MD simulations. Y.Z., Z.W., and X.W. contributed to CVD growth, TEM characterization, and data analysis. M.C. and R.L. wrote the paper. All the authors contributed to paper preparation and discussion.

\section{Competing interests}

The authors declare no competing interests. 


\section{Additional information}

Supplementary information is available for this paper at https://doi.org/10.1038/s41467020-14753-8.

Correspondence and requests for materials should be addressed to P.L. or M.C.

Peer review information Nature Communications thanks Andrea Li Bassi, Xinmao Yin and the other, anonymous, reviewer(s) for their contribution to the peer review of this work.

Reprints and permission information is available at http://www.nature.com/reprints

Publisher's note Springer Nature remains neutral with regard to jurisdictional claims in published maps and institutional affiliations. (c) (i) Open Access This article is licensed under a Creative Commons Attribution 4.0 International License, which permits use, sharing, adaptation, distribution and reproduction in any medium or format, as long as you give appropriate credit to the original author(s) and the source, provide a link to the Creative Commons license, and indicate if changes were made. The images or other third party material in this article are included in the article's Creative Commons license, unless indicated otherwise in a credit line to the material. If material is not included in the article's Creative Commons license and your intended use is not permitted by statutory regulation or exceeds the permitted use, you will need to obtain permission directly from the copyright holder. To view a copy of this license, visit http://creativecommons.org/ licenses/by/4.0/.

(C) The Author(s) 2020 